\title{
4-year Longitudinal Study of the Assessment of Body Posture, Back Pain, Postural and Life Habits of Schoolchildren
}

\author{
Bruna Nichele da Rosa ${ }^{1}$, Tássia Silveira Furlanetto ${ }^{1 *}$, Matias Noll2, Juliana Adami Sedrez ${ }^{1}$, \\ Emanuelle Francine Detogni Schmit ${ }^{1}$, Cláudia Tarragô Candotti ${ }^{1}$
}

\begin{abstract}
The aim of this study was to evaluate the behaviour of postural variables and the associated factors, like back pain and life habits, in schoolchildren during a period of four years. Forty-two school children underwent a postural assessment using Digital Image-Based Postural Assessment software. They also answered the self-administered questionnaire titled, "Back Pain and Body Posture Evaluation Instrument" to evaluate their back pain and life habits. We observed a significant trend of forward head, thoracic hyper kyphosis, and lumbar hyper lordosis in the entire group, as well as a trend of cervical rectification in girls. Both boys and girls presented a constant frequency in back pain, but girls presented higher prevalence along the years. We found a high prevalence of poor sitting posture in male (between 72.2-89.5\%) and female (between 73.3-100\%). We also found that girls had poor posture due to the way they carried their school supplies. However, occurred a decrease of time spent in front of television for male (from $87 \%$ to $10.5 \%$ ) and female (from $93.3 \%$ to $0 \%$ ). Significant changes occurred in analysed variables over evaluation period. Keywords: Back Pain, Posture, Adolescent, Child, Longitudinal Studies
\end{abstract}

\section{INTRODUCTION}

The Posture is a major factor in the health of the musculoskeletal system. Postural changes are related to painful and debilitating conditions (Ferreira, Duarte, Maldonado, Burke, \& Marques, 2010; McEvoy \& Grimmer, 2005). Back pain, specifically, is a common problem in younger schoolchildren, reaching up to $60 \%$ of this population in several countries (Ayanniyi, Mbada, \& Muolokwu, 2011; Calvo-Muñoz, Gómez-Conesa, \& Sánchez-Meca, 2013; Martínez-Crespo et al., 2009).

It is well documented that spine injuries and postural changes are results of genetics, age, and habits adopted throughout life (Adams \& Dolan, 2005; Giusti, De Almeida Jr, \& Tomasi, 2008). Children and teenagers are exposed to several risk factors like: low frequency in physical activities, extended periods in sedentary activities like watching television and playing videogames, carrying heavy backpacks improperly, and staying in a sitting position for long periods in inadequate furniture (Burton et al., 2005; Fraile García, 2009; Noll et al., 2013; Sedrez et al., 2014).

The developing musculoskeletal system presents particular characteristics and temporary postural alignment (Cil et al., 2005; Dimeglio, 2001) that lead to dynamic changes in postural alignment while a child grows (Cil et al., 2005; Schlösser, Vincken, Rogers, Castelein, \& Shah, 2015). There are constant changes in posture in children during growth spurt. Teenagers and adults, however present a predefined posture standard (Cil et al., 2005; Kobayashi, Atsuta, Matsuno, \& Takeda, 2004; Schlösser et al., 2015).

Therefore, it would seem appropriate to give special attention to children and teenagers, because their postural standard in adulthood is based on those stages of growth (Martelli \& Traebert, 2006). There are several studies describing postural standards in Brazilian schoolchildren (Baroni et al., 2015; Noll, Rosa, Candotti, Furlanetto, \& Gontijo, 2012; Sedrez et al., 2014). Even if we accept that body posture is not static, it tends to modify itself over the time,

\footnotetext{
Manuscript received at May $5^{\text {th }} 2016$; Accepted at June $25^{\text {th }} 2016$

${ }^{1}$ Universidade Federal do Rio Grande do Sul, Porto Alegre, Brazil.

${ }^{2}$ Instituto Federal Goiano, Goiânia, Brazil.

* Corresponding author: Av. Paulo Gama, 110 - Farroupilha, Porto Alegre - RS, 90040-060, Brasil

E-mail: tassiasf@gmail.com
} 
mainly during growth (Cil et al., 2005; Leboeuf, Letellier, Alos, Edery, \& Moldovan, 2009). The studies with children and teenagers are transversal, and do not provide evidence of how body posture behaves over the time. It was not found longitudinal studies with this age group in Brazilian population about the body posture behaviour. Therefore, the aim of this study was to evaluate the behaviour of postural variables and the associated factors, like back pain and life habits, in schoolchildren during a period of four years.

\section{METHOD}

This was a longitudinal study. The assessments always occurred in October during the four years evaluation: 2011, 2012, 2013 and 2014. The study occurred in Teutônia, a city of German colonization, located in the central region of Rio Grande do Sul, Brazil. We chose this area to develop this study because it is a small town, facilitating our access to the entire school population.

\section{Sample}

To select the sample, we randomly chose one out of eleven schools in Teutônia for the location of the study. Then, schoolchildren were chosen at random to participate.

In the first two years of assessment, we randomized the schoolchildren based on attendance list from formal physical education classes from $5^{\text {th }}$ to $8^{\text {th }}$ grade. In the following years, only those schoolchildren who participated to preliminary assessment were evaluated again.

The sample size was calculated based on the student population at the elementary school $(\mathrm{N}=1720)$, with a significance level of $95 \%$, and a sample error of $10 \%$ over the thoracic hyperkyphosis prevalence of $10 \%$ (Oshiro, Gabriele, \& Costa, 2007), which lead to the requirement of 34 student participants. Foreseeing a sample loss of $30 \%$, we invited 45 schoolchildren to participate. The inclusion criteria were: be enrolled between $5^{\text {th }}$ and $8^{\text {th }}$ grade in the first-year assessment; to attend at least three assessments; to have the ability to stay upright without assistance; and to have permission from their guardians to participate.
The exclusion criteria included anyone with acute musculoskeletal injury. This study was approved by the Ethics and Search Committee of Universidade Federal do Rio Grande do Sul, by number 19832, and respects the Resolution $466 / 2012$ of the Health National Council.

\section{Instruments}

The schoolchildren were evaluated using two instruments: (1) the Digital Imaged-based Postural Assessment (DIPA) by photogrammetry (Furlanetto, Candotti, Comerlato, \& Loss, 2012) and (2) the questionnaire titled "Back Pain and Body Posture Evaluation Instrument" (BackPEI) (Noll, Candotti, Vieira, \& Loss, 2013). In all four evaluations we randomized the order in which the tests were given (Photogrammetry and questionnaire).

\section{Procedures}

The postural assessment protocol consists of palpation and marking anatomic points (AP) for reference, digital photography registration, and image digitalization in DIPA. The same evaluators performed all analyses, in all four years. In this evaluation, schoolchildren wore swimsuits, were barefoot, and had their hair tied back if necessary.

We marked the following AP before getting the images: (1) on the sagittal plane - tragus, acromion, posterior superior iliac spine (PSIS), anterior superior iliac spine (ASIS), greater trochanter, lateral condyle of the knee, lateral malleolus and spinous process of C7, T6, L4 and S2 vertebrae; and (2) on the frontal plane acromion, inferior angle of scapula, PSIS and heels, all marked bilaterally, and spinous process of C7, T2, T4, T6, T8, T10, T12, L2, L4 and S2, according the protocol proposed by Furlanetto et al. (2012).

The schoolchildren were photographed in orthostatic, both (1) on the sagittal plane, in right side view, for assessment of anteroposterior changes, and (2) on the frontal plane, in back view, for assessment of lateral changes. After the photography registration, we transferred the images to a computer to digitalize them and then analyse them with the DIPA software (Furlanetto et al., 2012), that provided quantitative 
information about the students' posture as posture classification. Figures 1 and 2 represent the results from the DIPA, the variable used in this study, and its definition, on the frontal and sagittal planes.
Although DIPA software represents a classification for each evaluated body region, for analysis in this study, we grouped the posture classification into two categories: normal posture or postural change.
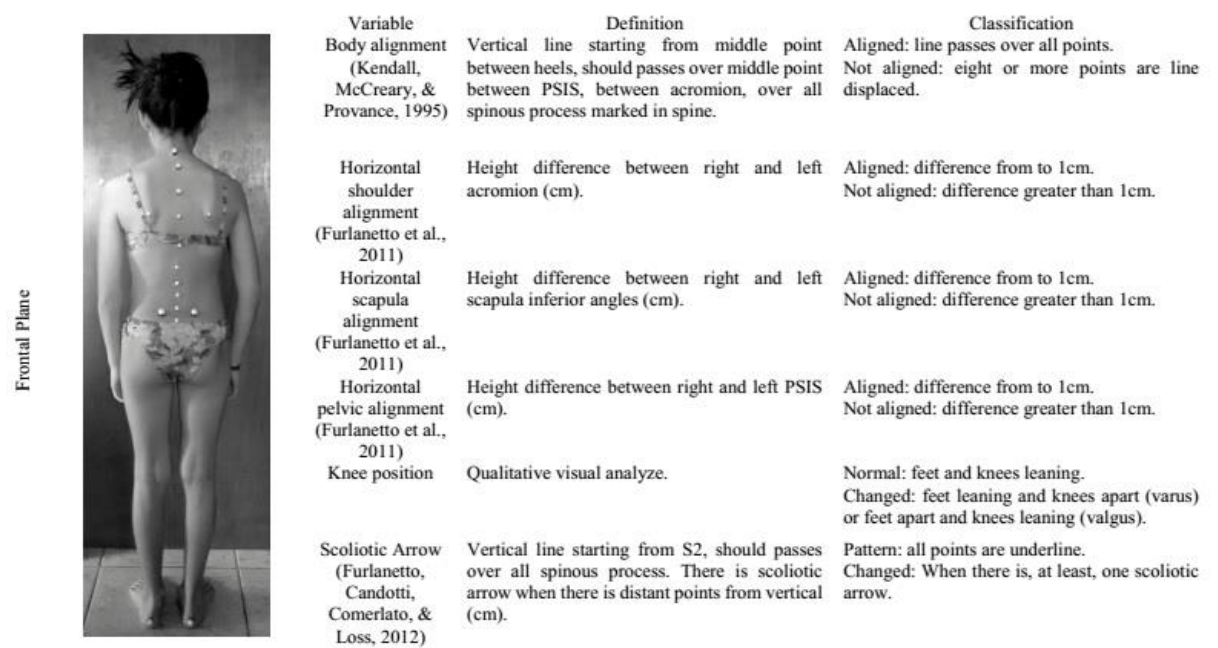

Figure 1. Results and variables provided by DIPA software, definitions and classifications in frontal plane
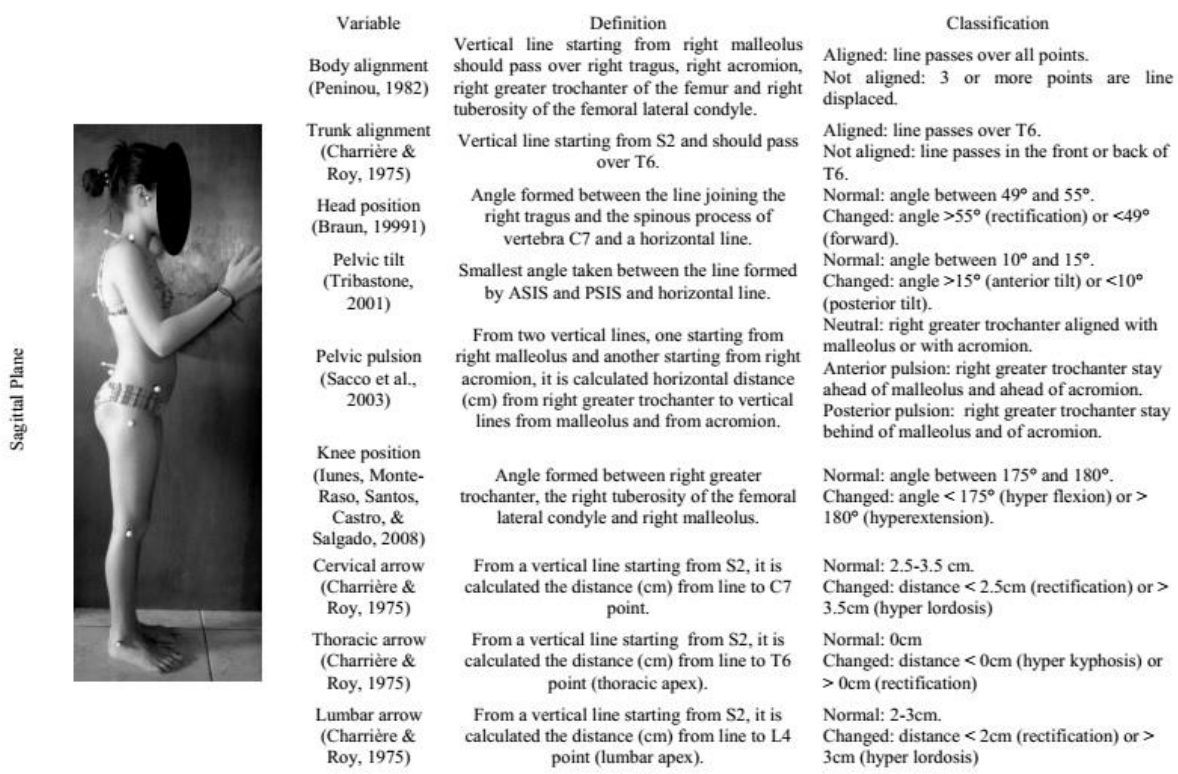

Figure 2. Results and variables provided by DIPA software, definitions and classifications in sagittal plane.

BackPEI (Noll, Candotti, Vieira, \& Loss, 2013) is an auto-applied questionnaire that is valid and reproducible, consists of 21 multiple choice questions, and has different versions for boys and girls. The questionnaire aims to identify the presence of back pain in the previous three months and evaluate demographic and behavioural risk factors. In this study, we used the questions to evaluate: (1) the presence of back pain (question 18); (2) the amount of physical exercise (question 1); (3) time spent watching television (question 4) and using the computer (question 5); and (4) postures adopted for writing in the classroom (question 9), or using the computer (question 11), how one carries a backpack (question 14) and one's sitting 
posture to talk (question 10). For analysis, we codify and tabulate the students' answers. In each question related to postural habits, only one indicates the adequate postural habit. The rest are grouped as inadequate postural habits.

\section{Statistical Analysis}

For statistical analysis, we used SPSS software version 18.0. The Shapiro Wilk test was used to verify the scalar variables' normality as provided from the DIPA. The scalar variables' descriptive statistic is shown by mean and standard deviation; and the categorical variables' description is by occurrence frequency of postural changes and habits collected over four years. For inferential analysis, we used repeated measures ANOVA with Bonferroni's Post-Hoc to verify scalar variables' differences over four years. For categorical variables, the differences over four years of evaluation were verified using the Friedman test with Wilcoxon Post-Hoc test. The significance level used was 0.05 .

The scalar variables used in this study was the gross value of horizontal shoulder alignment; horizontal scapula alignment; horizontal pelvic alignment; head position angle; pelvic angle; knee angle; cervical arrow; thoracic arrow; and lumbar arrow. The categorical variables included the postural and the behavioural variables. The postural variables were the classification of body alignment (on the sagittal and frontal plane); horizontal shoulder alignment; horizontal scapula alignment; pelvic alignment; knee position (on sagittal and frontal plane); scoliosis; trunk alignment (on sagittal plane); head position; pelvic position; pelvic pulsion; cervical spine; thoracic spine; lumbar spine. The behavioural variables were the classification of physical exercise practice; time spend watching TV per day; time spend using computer; sit posture to write; sit posture to talk; sit posture to use the computer; posture to carry backpack; and presence of back pain.

\section{RESULTS}

During the four years of evaluation, we assessed 45 schoolchildren, and 42 fulfilled the study inclusion criteria. The students were $59.5 \%$ $(n=25)$ male and $40.5 \%(n=17)$ female. Table 1 presents sample description.

Table 1

Sample anthropometric data (mean $\pm S D)$ in each evaluation year.

\begin{tabular}{|c|c|c|c|c|c|c|c|c|}
\hline \multirow{2}{*}{ Variable } & \multicolumn{2}{|c|}{ Year $1(n=42)$} & \multicolumn{2}{|c|}{ Year $2(n=34)$} & \multicolumn{2}{|c|}{ Year $3(n=37)$} & \multicolumn{2}{|c|}{ Year $4(n=37)$} \\
\hline & $\begin{array}{l}\text { Female } \\
(\mathrm{n}=17)\end{array}$ & $\begin{array}{c}\text { Male } \\
(n=25)\end{array}$ & $\begin{array}{l}\text { Female } \\
(n=10)\end{array}$ & $\begin{array}{c}\text { Male } \\
(n=24)\end{array}$ & $\begin{array}{l}\text { Female } \\
(\mathrm{n}=17)\end{array}$ & $\begin{array}{c}\text { Male } \\
(n=20)\end{array}$ & $\begin{array}{l}\text { Female } \\
(\mathrm{n}=16)\end{array}$ & $\begin{array}{c}\text { Male } \\
(n=21)\end{array}$ \\
\hline Age (year) & $13.2 \pm 1.1$ & $13 \pm 1.5$ & $14 \pm 1.2$ & $14 \pm 0.9$ & $14.8 \pm 0.9$ & $14.4 \pm 1.2$ & $15.8 \pm 1.2$ & $16.1 \pm 1.1$ \\
\hline Total & \multicolumn{2}{|c|}{$13.1 \pm 1.3$} & \multicolumn{2}{|c|}{$14 \pm 1.0$} & \multicolumn{2}{|c|}{$14.5 \pm 1.1$} & \multicolumn{2}{|c|}{$16 \pm 1.2$} \\
\hline Body mass (kg) & $52.3 \pm 13.2$ & $53.4 \pm 15.1$ & $59.2 \pm 19.9$ & $54.3 \pm 15.9$ & $55.2 \pm 7.5$ & $63.4 \pm 17.4$ & $57.1 \pm 9.5$ & $69.1 \pm 17.7$ \\
\hline Total & \multicolumn{2}{|c|}{$53 \pm 14.3$} & \multicolumn{2}{|c|}{$55.9 \pm 17.1$} & \multicolumn{2}{|c|}{$59.5 \pm 14.1$} & \multicolumn{2}{|c|}{$65.5 \pm 18.1$} \\
\hline Stature $(\mathrm{m})$ & $1.64 \pm 0.1$ & $1.58 \pm 0.1$ & $1.63 \pm 0.1$ & $1.64 \pm 0.1$ & $1.67 \pm 0.1$ & $1.72 \pm 0.1$ & $1.66 \pm 0.1$ & $1.78 \pm 0.1$ \\
\hline Total & \multicolumn{2}{|c|}{$1.6 \pm 0.1$} & \multicolumn{2}{|c|}{$1.63 \pm 0.1$} & \multicolumn{2}{|c|}{$1.7 \pm 0.1$} & \multicolumn{2}{|c|}{$1.72 \pm 0.1$} \\
\hline
\end{tabular}

In the scalar variable analysis (Table 2), all the variables on the frontal plane, pelvis angle on the sagittal plane and cervical arrow in girls did not present significant statistical differences $(\mathrm{p} \leq 0.05)$ during four years of evaluation and presented a similar behaviour between genders. A similar behaviour between boys and girls could also be observed in some variables in the sagittal plane: lumbar arrow, which presented a significant tendency from hyperlordosis to lumbar rectification posture; thoracic arrow, which showed a significant tendency of kyphosis increase; and a significant forward head position, over the four years of evaluation (Table 2).

However, in other variables on the sagittal plane (Table 2) there were different behaviours between genders over the four years. For example, in knee angle, boys presented a significant tendency to hyperflexion while girls presented within the normal range. The cervical arrow showed boys presented a posture oscillation, while girls presented a rectification tendency. 
Table 2

Mean \pm standard deviation of scalar postural variables in sagittal and frontal plane, among male $(M)$ and female $(F)$ gender.

\begin{tabular}{|c|c|c|c|c|c|c|c|}
\hline \multirow{7}{*}{ 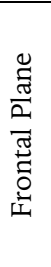 } & Variable & & Year 1 & Year 2 & Year 3 & Year 4 & Tendency \\
\hline & \multirow{2}{*}{$\begin{array}{c}\text { Horizontal shoulder } \\
\text { alignment }\end{array}$} & M & $1 \pm 0.7$ & $1.1 \pm 0.8$ & $0.9 \pm 0.8$ & $1 \pm 0.7$ & $\rightarrow$ \\
\hline & & $\mathrm{F}$ & $1.1 \pm 1$ & $0.7 \pm 0.7$ & $0.9 \pm 0.7$ & $0.9 \pm 0.7$ & $\rightarrow$ \\
\hline & \multirow{2}{*}{$\begin{array}{c}\text { Horizontal } \\
\text { scapula alignment }\end{array}$} & $\mathrm{M}$ & $0.7 \pm 0.5$ & $0.9 \pm 0.7$ & $0.9 \pm 0.7$ & $0.8 \pm 0.5$ & $\rightarrow$ \\
\hline & & $\mathrm{F}$ & $0.7 \pm 0.5$ & $0.6 \pm 0.7$ & $0.8 \pm 0.5$ & $0.6 \pm 0.4$ & $\rightarrow$ \\
\hline & \multirow{2}{*}{$\begin{array}{l}\text { Horizontal pelvic } \\
\text { alignment }\end{array}$} & $\mathrm{M}$ & $0.3 \pm 0.2$ & $0.3 \pm 0.3$ & $0.3 \pm 0.2$ & $0.4 \pm 0.4$ & $\rightarrow$ \\
\hline & & $\mathrm{F}$ & $0.4 \pm 0.3$ & $0.4 \pm 0.2$ & $0.3 \pm 0.2$ & $0.4 \pm 0.2$ & $\rightarrow$ \\
\hline \multirow{12}{*}{ 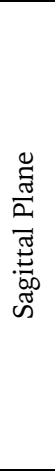 } & \multirow{2}{*}{ Head position } & $\mathrm{M}^{\mathrm{b}, \mathrm{c}}$ & $57.8 \pm 6$ & $53.6 \pm 6.4$ & $53.1 \pm 4.8$ & $47.8 \pm 3.4$ & From rectification to forward \\
\hline & & $\mathrm{F}^{\mathrm{d}}$ & $54.5 \pm 5.1$ & $51.6 \pm 4.3$ & $54.7 \pm 3.9$ & $49.7 \pm 4.9$ & From normal position to forward \\
\hline & \multirow{2}{*}{ Pelvic angle } & $\mathrm{M}$ & $11.4 \pm 4.3$ & $14.3 \pm 6.4$ & $12.9 \pm 6.6$ & $12.4 \pm 5.7$ & $\rightarrow$ \\
\hline & & $\mathrm{F}$ & $8.9 \pm 4.5$ & $10 \pm 5.1$ & $9.6 \pm 5$ & $11.3 \pm 5.1$ & $\rightarrow$ \\
\hline & \multirow{2}{*}{ Knee anlge } & $M^{\mathrm{d}, \mathrm{f}}$ & $176.3 \pm 5.7$ & $173.6 \pm 5.8$ & $178.3 \pm 5.5$ & $172.3 \pm 7.5$ & From normal position to hyper flexion \\
\hline & & $\mathrm{F}^{\mathrm{a}, \mathrm{c}, \mathrm{d}}$ & $182.8 \pm 5.4$ & $177.7 \pm 4.6$ & $181.3 \pm 5$ & $178.6 \pm 9.5$ & $\rightarrow$ \\
\hline & \multirow[t]{2}{*}{ Cervical arrow } & $\mathrm{M}^{\mathrm{a}, \mathrm{b}, \mathrm{e}, \mathrm{f}}$ & $5.1 \pm 2.4$ & $2.6 \pm 1.9$ & $1.6 \pm 2.1$ & $4.6 \pm 1.8$ & $\begin{array}{l}\text { From hyper lordosis to rectification until } \\
\text { year 3, returning to hyper lordosis }\end{array}$ \\
\hline & & $\mathrm{F}$ & $3.1 \pm 1.7$ & $1.9 \pm 1$ & $1.1 \pm 1.6$ & $2.4 \pm 1.6$ & Rectification \\
\hline & \multirow{2}{*}{ Thoracic arrow } & $\mathrm{M}^{\mathrm{b}, \mathrm{c}}$ & $1.1 \pm 1.6$ & $-1 \pm 1.6$ & $-2.5 \pm 2.3$ & $-2.3 \pm 1.8$ & From rectification to hyper kyphosis \\
\hline & & $\mathrm{F}^{c}$ & $-0.8 \pm 1.2$ & $-2 \pm 0.8$ & $-3.1 \pm 1.2$ & $-3.2 \pm 1.4$ & Increase of hyper kyphosis \\
\hline & \multirow{2}{*}{ Lumbar arrow } & $\mathrm{M}^{\mathrm{a}, \mathrm{b}, \mathrm{c}, \mathrm{d}, \mathrm{f}}$ & $3.4 \pm 0.8$ & $1.9 \pm 0.7$ & $0.9 \pm 0.7$ & $1.6 \pm 0.6$ & From hyper lordosis to rectification \\
\hline & & $\mathrm{F}^{\mathrm{a}, \mathrm{b}, \mathrm{c.d}, \mathrm{f}}$ & $3.4 \pm 0.7$ & $2.2 \pm 0.7$ & $1.6 \pm 0.8$ & $1.5 \pm 0.7$ & From hyper lordosis to rectification \\
\hline
\end{tabular}

${ }^{a}$ significant difference between Year 1 and Year $2(\mathrm{p} \leq 0.05) ;{ }^{\mathrm{b}}$ significant difference between Year 1 and Year $3(\mathrm{p} \leq 0.05)$; ${ }^{c}$ significant difference between Year 1 and Year $4(\mathrm{p} \leq 0.05)$; ${ }^{d}$ significant difference between Year 2 and Year 3 ( $\left.\mathrm{p} \leq 0.05\right)$; ${ }^{\mathrm{e}}$ significant difference between Year 2 and Year 4 ( $\left.\mathrm{p} \leq 0.05\right)$; ${ }^{\mathrm{f}}$ significant difference between Year 3 and Year $4(\mathrm{p} \leq 0.05)$; $\rightarrow$ Variable mean remained equal over the years

Table 3 shows the analysis of categorical variables over all four years. Only those variables that assess the spine posture on the sagittal plane showed different behaviour between boys and girls, but without significant differences. In boys, the cervical spine's prevalence of postural changes remained high. However, in girls repeated measures ANOVA shows a significant difference, but post-hoc was not sensitive enough to shows in which years occurred the difference. We observed a similar prevalence of postural changes until the third year of evaluation of girls' cervical spine, when there was a change: a decrease from the last year of evaluation. The prevalence of postural changes in thoracic spine among males remained high over the years, while in females we observed an increase in changes beginning with the second-year evaluation and continuing in the following years, but without significant differences. As for the lumbar spine, even without significant differences for boys and girls along the years, boys showed a prevalence of postural changes peak in the third-year evaluation. Among girls, the prevalence of postural changes stayed low until the third and fourth year evaluations, in which they presented high rates of postural changes.
The other variables presented a similar behaviour between boys and girls, continuing without significant differences over the evaluation years, except the pelvis pulsion in females, and the knee position and scoliosis in males. The pelvis pulsion presented a significant increase in prevalence of postural changes in females (Table 3). Both knee position and scoliosis in males presented a significant difference in repeated measures ANOVA, but the post-hoc was not sensitive enough to shows in which year occurred the difference.

Postural and life habits varied between males and females, like their computer habits, how they sat to talk and how they carried their backpacks (Table 4). Computer use among boys showed a decrease in the prevalence of inadequate habits over the time. The females presented an oscillation in behavior during the four evaluation years. The posture adopted to sit to talk presented a high prevalence of inadequate habits among boys and girls; however, boys' prevalence remained constant over the time, while girls experienced a significant increase in the second year of evaluation, reaching $100 \%$ prevalence, and staying that way throughout the study. 
Table 3

Percentage (frequency) of categorical postural variables on sagittal and frontal plane, among male $(M)$ and female $(F)$ genders.

\begin{tabular}{|c|c|c|c|c|c|c|c|c|}
\hline \multirow{25}{*}{ 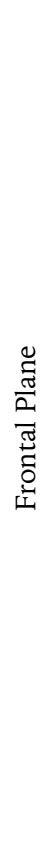 } & Variable & & Classification & Year 1 & Year 2 & Year 3 & Year 4 & Tendency \\
\hline & \multirow{4}{*}{$\begin{array}{c}\text { Body } \\
\text { alignment }\end{array}$} & \multirow{2}{*}{ M } & Normal & $30.4(7)$ & $18.2(4)$ & $45(9)$ & $9.5(2)$ & \multirow{2}{*}{$\rightarrow$} \\
\hline & & & Changed & $6.6(16)$ & $81.8(18)$ & $55(11)$ & $90.5(19)$ & \\
\hline & & \multirow{2}{*}{$\mathrm{F}$} & Normal & $35.7(5)$ & $30(3)$ & $17.6(3)$ & $18.8(3)$ & \multirow{2}{*}{$\rightarrow$} \\
\hline & & & Changed & $64.3(9)$ & $70(7)$ & $82.4(14)$ & $81.3(13)$ & \\
\hline & \multirow{4}{*}{$\begin{array}{l}\text { Horizontal } \\
\text { shoulder } \\
\text { alignment }\end{array}$} & \multirow{2}{*}{ M } & Normal & $52.2(12)$ & $54.5(12)$ & $55(11)$ & $57.1(12)$ & \multirow[b]{2}{*}{$\rightarrow$} \\
\hline & & & Changed & $47.8(11)$ & $45.5(10)$ & $45(9)$ & $42.9(9)$ & \\
\hline & & \multirow{2}{*}{$\mathrm{F}$} & Normal & 64.3(9) & $70(7)$ & $58.8(10)$ & $62.5(10)$ & \multirow[b]{2}{*}{$\rightarrow$} \\
\hline & & & Changed & $35.7(5)$ & $30(3)$ & $41.2(7)$ & $37.5(6)$ & \\
\hline & \multirow{4}{*}{$\begin{array}{c}\text { Horizontal } \\
\text { scapula } \\
\text { alignment }\end{array}$} & \multirow{2}{*}{ M } & Normal & 78.3(18) & $59.1(13)$ & $70(14)$ & $61.9(13)$ & \multirow{2}{*}{$\rightarrow$} \\
\hline & & & Changed & $21.7(5)$ & $40.9(9)$ & $30(6)$ & $38.1(8)$ & \\
\hline & & & Normal & $85.7(12)$ & $80(8)$ & $58.8(10)$ & $81.3(13)$ & \\
\hline & & $\mathrm{F}$ & Changed & $14.3(2)$ & $20(2)$ & $41.2(7)$ & $18.8(3)$ & $\rightarrow$ \\
\hline & & $M$ & Normal & $100(23)$ & $95.5(21)$ & $100(20)$ & $95.2(20)$ & \\
\hline & Pelvic & $M$ & Changed & $0(0)$ & $4.5(1)$ & $0(0)$ & $4.8(1)$ & $\rightarrow$ \\
\hline & alignment & $F_{1}$ & Normal & $100(14)$ & $100(10)$ & $100(17)$ & $100(16)$ & \\
\hline & & $\Gamma$ & Changed & $0(0)$ & $0(0)$ & $0(0)$ & $0(0)$ & $\rightarrow$ \\
\hline & & $M^{*}$ & Normal & $52.2(12)$ & $27.3(6)$ & $35(7)$ & 14.3(3) & \\
\hline & Knee & $\mathrm{M}^{*}$ & Changed & $47.8(11)$ & $72.7(16)$ & $65(13)$ & $85.7(18)$ & $\rightarrow$ \\
\hline & position & $\Gamma$ & Normal & $35.7(5)$ & $50(5)$ & $41.2(7)$ & $37.5(6)$ & \\
\hline & & $\mathrm{F}$ & Changed & $64.3(9)$ & $50(5)$ & $58.8(10)$ & $62.5(10)$ & $\rightarrow$ \\
\hline & & & Normal & 21.7(5) & $45.5(10)$ & $30(6)$ & $42.9(9)$ & \\
\hline & & $M^{*}$ & Changed & $78.3(18)$ & $54.5(12)$ & $70(14)$ & $57.1(12)$ & $\rightarrow$ \\
\hline & Scollosis & $F_{2}$ & Normal & $28.6(4)$ & $70(7)$ & $41.2(7)$ & $50(8)$ & \\
\hline & & $\mathrm{F}$ & Changed & 71.4(10) & $30(3)$ & $58.8(10)$ & $50(8)$ & $\rightarrow$ \\
\hline & & & Normal & $5.6(1)$ & $0(0)$ & $0(0)$ & $0(0)$ & \\
\hline & Body & $\mathrm{M}$ & Changed & $94.4(17)$ & $100(18)$ & $100(17)$ & $100(20)$ & $\rightarrow$ \\
\hline & alignment & $F_{1}$ & Normal & $0(0)$ & $0(0)$ & $0(0)$ & $6.3(1)$ & \\
\hline & & $\mathrm{F}$ & Changed & $100(12)$ & $100(9)$ & $100(17)$ & $9.8(15)$ & $\rightarrow$ \\
\hline & & $M$ & Normal & $27.8(5)$ & $5.6(1)$ & $5.9(1)$ & $35(7)$ & \\
\hline & Trunk & $\mathrm{M}$ & Changed & $72.2(13)$ & $94.4(17)$ & $94.1(16)$ & $65(13)$ & $\rightarrow$ \\
\hline & alignment & $\mathrm{F}$ & Normal & $8.3(1)$ & $0(0)$ & $5.9(1)$ & $18.8(3)$ & \\
\hline & & $\mathrm{F}$ & Changed & $91.7(11)$ & $100(9)$ & $94.1(16)$ & $81.3(13)$ & \\
\hline & & & Normal & $3.3(6)$ & $50(9)$ & $64.7(11)$ & $45(9)$ & $\rightarrow$ \\
\hline & Head & $\mathrm{M}$ & Changed & $66.7(12)$ & $50(9)$ & $35.3(6)$ & $55(11)$ & $\rightarrow$ \\
\hline & position & $\Gamma$ & Normal & 41.7(5) & $55.6(5)$ & $58.8(10)$ & $56.3(9)$ & \\
\hline & & $\mathrm{F}$ & Changed & $58.3(7)$ & $44.4(4)$ & $41.2(7)$ & $43.8(7)$ & $\rightarrow$ \\
\hline & & & Normal & $27.8(5)$ & $22.2(4)$ & $29.4(5)$ & $30(6)$ & \\
\hline & Pelvic & M & Changed & $72.2(13)$ & $77.8(14)$ & $70.6(12)$ & $70(14)$ & $\rightarrow$ \\
\hline & position & $\mathrm{F}$ & Normal & $16.7(2)$ & $33.3(3)$ & $41.2(7)$ & $37.5(6)$ & \\
\hline$\underset{\widetilde{\varpi}}{\mathscr{\Xi}}$ & & & Changed & $83.3(10)$ & $66.7(6)$ & $58.8(10)$ & $62.5(10)$ & $\rightarrow$ \\
\hline$\vec{a}$ & & $M$ & Normal & $50(9)$ & $38.9(7)$ & $47.1(8)$ & $0(0)$ & ( \\
\hline స్త్ర & Pelvic & $\mathrm{M}$ & Changed & $50(9)$ & $61.1(11)$ & $52.9(9)$ & $100(20)$ & $\rightarrow$ \\
\hline 5 & pulsion & $F^{c, e, f}$ & Normal & $50(6)$ & $55.6(5)$ & $23.5(4)$ & $0(0)$ & \\
\hline & & $\mathrm{F}^{\mathrm{T}, e, 1}$ & Changed & $50(6)$ & $44.4(4)$ & $76.5(13)$ & $100(16)$ & $\uparrow$ \\
\hline & & $M$ & Normal & $50(9)$ & $50(9)$ & $41.2(7)$ & $30(6)$ & \\
\hline & Knee & $M$ & Changed & $50(9)$ & $50(9)$ & $58.8(10)$ & $70(14)$ & $\rightarrow$ \\
\hline & position & $F_{0}$ & Normal & $16.7(2)$ & $44.4(4)$ & $35.3(6)$ & $37.5(6)$ & 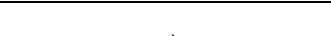 \\
\hline & & $\mathrm{F}$ & Changed & $83.3(10)$ & $55.6(5)$ & $64.7(11)$ & $62.5(10)$ & $\rightarrow$ \\
\hline & & $M$ & Normal & $22.2(4)$ & $33.3(6)$ & $23.5(4)$ & $25(5)$ & $\rightarrow$ \\
\hline & Cervical & $\mathrm{M}$ & Changed & $77.8(14)$ & $66.7(12)$ & $76.5(13)$ & $75(15)$ & $\rightarrow$ \\
\hline & spine & $E^{*}$ & Normal & $50(6)$ & $55.6(5)$ & $5.9(1)$ & $50(8)$ & 1 in wor 2 ond 1 in wor 4 \\
\hline & & $\mathrm{F}^{*}$ & Changed & $50(6)$ & $44.4(4)$ & $94.1(16)$ & $50(8)$ & $\uparrow$ in year 3 and $\downarrow$ in year 4 \\
\hline & & M & Normal & $55.6(10)$ & $44.4(8)$ & $11.8(2)$ & $35(7)$ & $\rightarrow$ \\
\hline & Thoracic & $\mathrm{M}$ & Changed & $44.4(8)$ & $55.6(10)$ & $88.2(15)$ & $65(13)$ & $\rightarrow$ \\
\hline & spine & $\mathrm{F}$ & Normal & $58.3(7)$ & $0(0)$ & $5.9(1)$ & $6.3(1)$ & $\uparrow$ in vear 2 and $\rightarrow$ \\
\hline & & $\mathrm{F}$ & Changed & $41.7(5)$ & $100(9)$ & $94.1(16)$ & $93.8(15)$ & $\uparrow$ in year 2 and $\rightarrow$ \\
\hline & & $M$ & Normal & $55.6(10)$ & $72.2(13)$ & $23.5(4)$ & $50(10)$ & $\uparrow$ in 2 \\
\hline & Lumbar & $\mathrm{M}$ & Changed & $44.4(8)$ & $27.8(5)$ & $76.5(13)$ & $50(10)$ & T in year 3 \\
\hline & spine & $F_{2}$ & Normal & $75(9)$ & $88.9(8)$ & $41.2(7)$ & $43.8(7)$ & $\uparrow$ in woar 2 \\
\hline & & $\mathrm{F}$ & Changed & $25(3)$ & $11.1(1)$ & $58.8(10)$ & $56.3(9)$ & $\uparrow$ in year 3 \\
\hline
\end{tabular}

${ }^{a}$ significant difference between Year 1 and Year $2(\mathrm{p} \leq 0.05)$; ${ }^{\mathrm{b}}$ significant difference between Year 1 and Year $3(\mathrm{p} \leq 0.05)$; ${ }^{c}$ significant difference between Year 1 and Year 4 ( $\left.\mathrm{p} \leq 0.05\right)$; ${ }^{d}$ significant difference between Year 2 and Year 3 ( $\left.\mathrm{p} \leq 0.05\right)$; ${ }^{\mathrm{e}}$ significant difference between Year 2 and Year 4 ( $\left.\mathrm{p} \leq 0.05\right)$; ${ }^{\mathrm{f}}$ significant difference between Year 3 and Year 4 ( $\left.\mathrm{p} \leq 0.05\right)$; $\rightarrow$ Variable change prevalence remained equal over the years; $\uparrow$ Variable change prevalence increased over the years; $\downarrow$ Variable change prevalence decreased over the years; *significant difference in repeated measures ANOVA 
When it came to carry a backpack, boys presented a decrease in inadequate habit prevalence in the second year of evaluation, remaining low for the remaining evaluation period. We observed a high prevalence in inadequate backpack habits during all four evaluation years among girls. Concerning to back pain, there was a high and constant prevalence of back pain among girls, though boys showed an oscillation in prevalence of back pain presence over the years, but without significant difference along the years.

\section{Table 4}

Percentage (frequency) of postural and life habits and back pain among male $(M)$ and female $(F)$ genders.

\begin{tabular}{|c|c|c|c|c|c|c|c|}
\hline Question & & Classification & Year 1 & Year 2 & Year 3 & Year 4 & Tendency \\
\hline \multirow{4}{*}{$\begin{array}{l}\text { Physical } \\
\text { exercise } \\
\text { practice }\end{array}$} & \multirow{2}{*}{ M } & Yes & $100(23)$ & $83.3(15)$ & $94.1(16)$ & $89.5(17)$ & \multirow{2}{*}{$\rightarrow$} \\
\hline & & No & $0(0)$ & $16.7(3)$ & $5.9(1)$ & $10.5(2)$ & \\
\hline & \multirow{2}{*}{$\mathrm{F}$} & Yes & $93.3(14)$ & $87.5(7)$ & $76.5(13)$ & $92.9(13)$ & \multirow{2}{*}{$\rightarrow$} \\
\hline & & No & $6.7(1)$ & $12.5(1)$ & $23.5(4)$ & $7.1(1)$ & \\
\hline \multirow{4}{*}{$\begin{array}{c}\text { Time spend } \\
\text { watching TV } \\
\text { per day }\end{array}$} & \multirow{2}{*}{$M^{a, b, c}$} & Adequate time $(0-3 \mathrm{~h})$ & $13(3)$ & $83.3(15)$ & $82.4(14)$ & $89.5(17)$ & \multirow{2}{*}{$\downarrow$ in year 2 and $\rightarrow$} \\
\hline & & Inadequate time $(>4 \mathrm{~h})$ & $87(20)$ & $16.7(3)$ & $17.6(3)$ & $10.5(2)$ & \\
\hline & \multirow{2}{*}{$\mathrm{F}^{\mathrm{b}, \mathrm{c}}$} & Adequate time $(0-3 \mathrm{~h})$ & $6.7(1)$ & $87.5(7)$ & $70.6(12)$ & $100(14)$ & \multirow{2}{*}{$\downarrow$ in year 2 and $\rightarrow$} \\
\hline & & Inadequate time $(>4 \mathrm{~h})$ & $93.3(14)$ & $12.5(1)$ & $29.4(5)$ & $0(0)$ & \\
\hline \multirow{4}{*}{$\begin{array}{l}\text { Time spend } \\
\text { using } \\
\text { computer }\end{array}$} & \multirow{2}{*}{$M^{a, b, c}$} & Adequate time $(0-3 \mathrm{~h})$ & $4.3(1)$ & $55.6(10)$ & $58.8(10)$ & $52.6(10)$ & \multirow{2}{*}{$\downarrow$ in year 2 and $\rightarrow$} \\
\hline & & Inadequate time $(>4 \mathrm{~h})$ & $95.7(22)$ & $44.4(8)$ & $41.2(7)$ & $47.4(9)$ & \\
\hline & \multirow{2}{*}{$\mathrm{F}^{\mathrm{c}, \mathrm{f}}$} & Adequate time $(0-3 \mathrm{~h})$ & $0(0)$ & $75(6)$ & $29.4(5)$ & $100(14)$ & \multirow{2}{*}{$\begin{array}{c}\downarrow \text { in year } 2 \text { and } \uparrow \text { in year } \\
3 \text { and } \downarrow \text { in year } 4\end{array}$} \\
\hline & & Inadequate time $(>4 \mathrm{~h})$ & $100(15)$ & $25(2)$ & $70.6(12)$ & $0(0)$ & \\
\hline \multirow{4}{*}{$\begin{array}{l}\text { Sit posture to } \\
\text { write }\end{array}$} & \multirow{2}{*}{ M } & Adequate & $17.4(4)$ & $16.7(3)$ & $11.8(2)$ & $15.8(3)$ & \multirow[b]{2}{*}{$\rightarrow$} \\
\hline & & Inadequate & $82.6(19)$ & $83.3(15)$ & $88.2(15)$ & $84.2(16)$ & \\
\hline & \multirow{2}{*}{$\mathrm{F}$} & Adequate & $6.7(1)$ & $0(0)$ & $0(0)$ & $0(0)$ & \multirow{2}{*}{$\rightarrow$} \\
\hline & & Inadequate & $93.3(14)$ & $100(8)$ & $100(17)$ & $100(14)$ & \\
\hline \multirow{4}{*}{$\begin{array}{l}\text { Sit posture to } \\
\text { talk }^{\mathrm{b}, \mathrm{c}}\end{array}$} & \multirow{2}{*}{ M } & Adequate & $21.7(5)$ & $11.1(2)$ & $11.8(2)$ & $10.5(2)$ & \multirow[b]{2}{*}{$\rightarrow$} \\
\hline & & Inadequate & $78.3(18)$ & $88.9(16)$ & $88.2(15)$ & $89.5(17)$ & \\
\hline & \multirow{2}{*}{$\mathrm{F}^{\mathrm{b}}$} & Adequate & $26.7(4)$ & $0(0)$ & $0(0)$ & $0(0)$ & \multirow{2}{*}{$\uparrow$ in year 2 and $\rightarrow$} \\
\hline & & Inadequate & $73.3(11)$ & $100(8)$ & $100(17)$ & $100(14)$ & \\
\hline \multirow{4}{*}{$\begin{array}{l}\text { Sit posture to } \\
\text { use computer }\end{array}$} & \multirow{2}{*}{ M } & Adequate & $13(3)$ & $27.8(5)$ & $23.5(4)$ & $10.5(2)$ & \multirow{2}{*}{$\rightarrow$} \\
\hline & & Inadequate & $87(20)$ & $72.2(13)$ & $76.5(13)$ & $89.5(17)$ & \\
\hline & \multirow[b]{2}{*}{$\mathrm{F}$} & Adequate & $0(0)$ & $0(0)$ & $0(0)$ & $0(0)$ & \\
\hline & & Inadequate & $100(15)$ & $100(8)$ & $100(17)$ & $100(14)$ & $\rightarrow$ \\
\hline & & Adequate & $43.5(10)$ & $94.4(17)$ & $82.4(14)$ & $94.7(18)$ & \\
\hline Posture to & $\mathrm{M}^{\mathrm{a}, \mathrm{c}}$ & Inadequate & $56.5(13)$ & $5.6(1)$ & $17.6(3)$ & $5.3(1)$ & $\downarrow$ in year 2 and $\rightarrow$ \\
\hline $\begin{array}{c}\text { carry } \\
\text { backnack }\end{array}$ & & Adequate & $33.3(5)$ & $37.5(3)$ & $17.6(3)$ & $35.7(5)$ & \\
\hline & $\mathrm{F}$ & Inadequate & $66.7(10)$ & $62.5(5)$ & $82.4(14)$ & $64.3(9)$ & $\rightarrow$ \\
\hline & & Yes & $39.1(9)$ & $55.6(10)$ & $23.5(4)$ & $63.2(12)$ & \\
\hline Presence of & $\mathrm{M}$ & No & $60.9(14)$ & $44.4(8)$ & $76.5(13)$ & $36.8(7)$ & $\rightarrow$ \\
\hline back pain & & Yes & $60(9)$ & $75(6)$ & $64.7(11)$ & $78.6(11)$ & \\
\hline & $\mathrm{F}$ & No & $40(4)$ & $25(2)$ & $35.3(6)$ & $21.4(3)$ & $\rightarrow$ \\
\hline
\end{tabular}

\section{DISCUSSION}

Over the four years evaluation period, we observed some similar behaviours between male and female posture. For example, a tendency to experience lumbar rectification, a thoracic hyperkyphosis, and a forward head. These tendencies might be the results of postural habits adopted over time, once we observed high prevalence of inadequate habits in sitting position, both boys and girls, like, posture adopted to write, to sit to talk and to use computer.

We know postural and behavioural habits are identified as risk factors for changes in posture.
(Balagué et al., 1999; Fraile García, 2009; Sedrez et al., 2014). In a sitting posture, it is documented that slouching increases the lumbar flexion and posterior pelvic tilt compared to sitting erect; the same occurs comparing cross-legged sitting and sitting erect (Yu \& An, 2015). The lumbar flexion and posterior pelvic tilt are more evident when associated with the two postures, and especially when the subject adopts the slouched crosslegged sitting position (Yu \& An, 2015).

These sitting postures are the most used among people ( $\mathrm{Yu} \& \mathrm{An}, 2015)$, mainly in a school environment (Noll et al., 2013) since this population spends up to seven hours a day in the 
sitting position (Fraile García, 2009). In addition, we observed that the erect sitting posture is hard to adopt during long periods, since it requires balance and muscle strength (Yu \& An, 2015). We found a high prevalence of inadequate postural habits of sit to talk in boys consistently over all four years. In girls, we found $100 \%$ of inadequate habits in year two of the study. The slouched sitting position still affects the thoracic spine, which causes a significant increase of thoracic flexion (O'Sullivan et al., 2006) and a possible association to forward head (Caneiro et al., 2010).

Another postural habit that showed a high prevalence of inadequate posture was the sitting posture adopted for using a computer, which is aggravated because of the time spent doing so. Although girls showed an oscillation in prevalence of inadequate habits reaching zero in the last evaluation year, and boys had decreased the prevalence of this inadequate habit, a large portion of the study showed time using the computer averaged over 4 hours every day. Sitting for a long time leads to many risk factors that cause postural changes (O'Sullivan et al., 2006; Yu \& An, 2015). Those risks are exacerbated by computer use, which has already been identified as predisposed to musculoskeletal diseases, mainly in the upper limbs and cervical spine (Marcus et al., 2002).

Concerning to prevalence of postural changes, we observed significant differences in cervical spine and pelvic pulsion on sagittal plane, both for girls and in knee position and scoliosis on frontal plane, both for boys. However, the thoracic and lumbar spine presented an increase in changes from the second and third years, reaching the prevalence of $100 \%$ of change in thoracic spine in girls on second year evaluation, but without significant differences in both variables. During the second and third year, girls presented fourteen years old on average, about two or three years later beginning of growth spurt (Contri, Petrucelli, \& Perea, 2009). The average time of a growth spurt is three years, and the first two years are the acceleration phase. Growth reaches its peak after the first two years, then the pace of growth starts to slow down (Castilho \& Filho, 2000). This growth spurt happens at the average age of fourteen. It is associated with the appearance of several postural changes, mainly due to the body balancing itself to be compatible to new body proportions (Contri et al., 2009). This might explain the increase in prevalence of postural changes among girls during the second and third evaluation years. Girls present a higher chance to develop postural changes during this age, compared to boys, since the growth spurt period coincides with the increase of circulating hormones. This includes estrogen, which interacts with growth hormones and other growth factors like bone acquisition, which is considered a potential etiological factor for postural changes (Leboeuf et al., 2009). However, both boys and girls presented an oscillation in scoliosis prevalence, while in boys there were significant differences according to repeated measures ANOVA, and in a general, boys presented slightly higher prevalence comparing to girls. This finding is interesting once the literature shows the female gender is an associated factor to scoliosis (Baroni et al., 2015; Burgoyne \& Fairbank, 2001), but there are indications in literature of similar behaviour of scoliosis in boys and girls (Nery, Halpern, Nery, Nehme, \& Stein, 2010; Rocha, Tatmatsu, \& Vilela, 2012).

Another important finding was the high prevalence of back pain among the assessed population. Both boys and girls presented constant prevalence of back pain without significant differences, but boys presented smaller prevalence compared to girls. Girls presented a constant prevalence of back pain from $60 \%$ and $78 \%$ over the term of the study. Studies have shown the youth population, both Brazilian and foreign, is affected by high prevalence of back pain (Noll et al., 2013; Skoffer, 2007). Back pain among this population is already considered a public health problem. It increases over the years and can be associated with other disorders like sleep disturbances, as well as the need for specialized medical care and medication (Ayanniyi, Mbada, \& Muolokwu, 2011; Balagué et al., 1999; Calvo-Muñoz, Gómez-Conesa, \& Sánchez-Meca, 2013; Skoffer, 2007).

As for risk factors for development of back pain, we found that females present higher 
prevalence than males (Balagué et al., 1999; Hoy, Brooks, Blyth, \& Buchbinder, 2010). Habits like time spent watching television (Balagué et al., 1999; Noll et al., 2013), staying in a sitting position, adopting an inadequate sitting posture, or carrying a backpack in an asymmetric way (Korovessis, Koureas, Zacharatos, \& Papazisis, 2005) also are risk factors to back pain development. We also observed a different behaviour between boys and girls when it came to carry a backpack. We found decreasing inadequate habits prevalence among males and an increase of prevalence over the years for females.

This study only presents an outlook of the variables in schoolchildren and does not present a relationship between analysed variables. Because of this, we suggest future studies to determine whether there is any relationship between body posture, back pain and habits. The importance of preventive actions in a school environment is evident. These habits mean the potential for future problems like postural changes and back pain (Balagué et al., 1999; Fraile García, 2009; Noll, et al., 2013; Sedrez et al., 2014) and can prevent the premature acquisition of inadequate habits among this population. The back-school methodology demonstrates a viable alternative, since raising awareness about back care and how best to perform daily activities has been shown to be effective among this group. (Fonseca et al., 2015; Noll, Candotti, \& Vieira, 2012).

\section{CONCLUSION}

In our four years of observations, we saw several changes, in both postural patterns and postural and life habits among schoolchildren. There was a similar tendency for a forward head, an increase of thoracic kyphosis, and lumbar rectification between both boys and girls. We observed high and constant prevalence of postural changes in cervical spine and thoracic spine among boys with a peak of changes in lumbar spine among this group in the third evaluation year. Girls presented significant increase in prevalence of postural changes in the cervical spine and lumbar spine in the third evaluation year and in the thoracic spine in the second evaluation year.

We observed a high frequency of back pain, occurring oscillation in prevalence of back pain among boys, while girls presented high and constant prevalence of back pain over the years. Furthermore, we observed high frequency in inadequate habits in sitting posture between both genders, like posture sitting to talk, to write and to use the computer. We also observed a high prevalence of inadequate posture for carrying backpacks among girls, an oscillation in time spent using the computer among girls and a decrease in this prevalence among boys. The habit of spending time watching television decreased over the years among both boys and girls.

We conclude there should be more health education for students about poor habits leading to back pain. We suggest teaching practical habits that promote the correct ways to carry oneself and avoid spine injuries and postural changes. This awareness effort could easily be included in Back School programs.

Acknowledgments:

To CNPQ for financial support for this research

Conflict of interests:

Nothing to declare.

\begin{tabular}{l}
\hline Funding: \\
CNPQ Internship with intern announcement pro \\
research 2012 of Propesq UFRGS \\
\hline
\end{tabular}

\section{REFERENCES}

Adams, M., \& Dolan, P. (2005). Spine biomechanics. Journal of Biomechanics, 38(10), 1972-1983. http://doi.org/10.1016/j.jbiomech.2005.03.028

Ayanniyi, O., Mbada, C. E., \& Muolokwu, C. A. (2011). Prevalence and profile of back pain in Nigerian adolescents. Medical Principles and Practice, 20(4), 368-373. http://doi.org/10.1159/000323766

Baroni, M. P., José, G., Sanchis, B., José, S., Assis, C. De, Gomes, R., ... Lopes, J. M. (2015). Factors Associated With Scoliosis in Schoolchildren: a Cross-Sectional Population-Based Study. Journal of Epidemiology, 25(3), 212-220. http://doi.org/10.2188/jea.JE20140061

Burgoyne, W., \& Fairbank, J. (2001). The management of scoliosis. Current Paediatrics, 11(5), 323-331. https://doi.org/10.1054/cupe.2001.0221 
Burton, A. K., Balagué, F., Cardon, G., Eriksen, H. R., Hänninen, O., Harvey, E., ... van der Beek, A. (2005). How to prevent low back pain. Best Practice and Research: Clinical Rheumatology, 19(4), 541-555. http://doi.org/10.1016/j.berh.2005.03.001

Calvo-Muñoz, I., Gómez-Conesa, A., \& Sánchez-Meca, J. (2013). Prevalence of low back pain in children and adolescents: a meta-analysis. BMC Pediatrics, 13(1), 14. http://doi.org/10.1186/1471-2431$13-14$

Cil, A., Yazici, M., Uzumcugil, A., Kandemir, U., Alanay, A., Alanay, Y., ... Surat, A. (2005). The evolution of sagittal segmental alignment of the spine during childhood. Spine, 30(1), 93-100. http://doi.org/10.1097/01.brs.0000149074.2155 0.32

Dimeglio, A. (2001). Growth in Pediatric Orthopaedics. Journal of Pediatric Orthopedics, 21, 549-555.

Ferreira, E. A. G., Duarte, M., Maldonado, E. P., Burke, T. N., \& Marques, A. P. (2010). Postural assessment software (PAS/SAPO): Validation and reliability. Clinics, 65(7), 675-81. http://doi.org/10.1590/S180759322010000700005

Fraile García, P. A. (2009). Dolor de espalda en alumnos de primaria y sus causas. Fisioterapia, $31(4)$, 137-142. http://doi.org/10.1016/j.ft.2008.04.001

Furlanetto, T. S., Candotti, C. T., Comerlato, T., \& Loss, J. F. (2012). Validating a postural evaluation method developed using a Digital Image-based Postural Assessment (DIPA) software. Computer Methods and Programs in Biomedicine, 108(1), 203-212. http://doi.org/10.1016/j.cmpb.2012.03.012

Giusti, P. H., De Almeida Jr., H. L., \& Tomasi, E. (2008). Weight excess of school materials and its risks factors in South Brazil. European Journal of Physical and Rehabilitation Medicine, 44(1), 33-38.

Kobayashi, T., Atsuta, Y., Matsuno, T., \& Takeda, N. (2004). A Longitudinal Study of Congruent Sagittal Spinal Alignment in an Adult Cohort. Spine, 29(6), 671-676.

Leboeuf, D., Letellier, K., Alos, N., Edery, P., \& Moldovan, F. (2009). Do estrogens impact adolescent idiopathic scoliosis? Trends in Endocrinology and Metabolism, 20(4), 147-152. http://doi.org/10.1016/j.tem.2008.12.004

Martelli, R. C., \& Traebert, J. (2006). Estudo descritivo das alterações posturais de coluna vertebral em escolares de 10 a 16 anos de idade: Tangará-SC, 2004. Revista Brasileira de Epidemiologia, 9(1), 87-
93. 790X2006000100011

http://doi.org/10.1590/S1415-

Martínez-Crespo, G., Rodríguez-Piñero Durán, M., López-Salguero, A. I., Zarco-Periñan, M. J., Ibáñez-Campos, T., \& Echevarría-Ruiz de Vargas, C. (2009). Dolor de espalda en adolescentes: prevalencia y factores asociados. Rehabilitación, 43(2), 72-80. http://doi.org/10.1016/S00487120(09)70773-X

McEvoy, M. P., \& Grimmer, K. (2005). Reliability of upright posture measurements in primary school children. BMC Musculoskeletal Disorders, 6, 35. http://doi.org/10.1186/1471-2474-6-35

Nery, L. S., Halpern, R., Nery, P. C., Nehme, K. P., \& Stein, A. T. (2010). Prevalence of scoliosis among school students in a town in southern Brazil. Sao Paulo Medical Journal, 128(2), 69-73. http://doi.org/10.1590/S151631802010000200005

Noll, M., Candotti, C. T., Rosa, B. N. da, Schoenell, M. C. W., Tiggemann, C. L., \& Loss, J. F. (2013). Back pain and the postural and behavioral habits of students in the municipal school network of Teutônia, Rio Grande do Sul. Journal of Human Growth and Development, 23(2), 129-135.

Noll, M., Candotti, C. T., Vieira, A., \& Loss, J. F. (2013). Back Pain and Body Posture Evaluation Instrument (BackPEI): development, content validation and reproducibility. International Journal of Public Health, 58(565-572). http://doi.org/10.1007/s00038-012-0434-1

Noll, M., Rosa, B. N. da, Candotti, C. T., Furlanetto, T. S., \& Gontijo, K. N. S. (2012). Alterações posturais em escolares do ensino fundamental de uma escola de Teutônia/RS. Revista Brasileira de Ciência E Movimento, 20(2), 32-42.

Rocha, J. C. T., Tatmatsu, D. I. B., \& Vilela, D. A. (2012). Associação entre uso de mochilas escolares e escoliose em adolescentes de escolas públicas e privadas. Motricidade, 8(S2), 803-809.

Schlösser, T. P. C., Vincken, K. L., Rogers, K., Castelein, R. M., \& Shah, S. A. (2015). Natural sagittal spino-pelvic alignment in boys and girls before, at and after the adolescent growth spurt. European Spine Journal, 24, 1158-1167. http://doi.org/10.1007/s00586-014-3536-z

Sedrez, J. A., Furlanetto, T. S., Noll, M., Gontijo, K. N. S., Rosa, B. N. Da, \& Candotti, C. T. (2014). Relação entre alterações posturais e fatores associados em escolares do ensino fundamental. Revista Baiana Saúde Pública, 38(2), 279-296. http://doi.org/10.5327/Z0100-02332014380200005

Tribastone, F. (2001). Tratado de exercícios corretivos: aplicados à reeducação postural. São Paulo: Manole.

All content of Journal Motricidade is licensed under Creative Commons, except when otherwise specified and in content retrieved from other bibliographic sources. 